
\title{
R Research Soure \\ Multiterminal Pathfinding in Practical VLSI Systems with Deep Neural Networks
}

Dmitry Utyamishev ( $\square$ dutyam2@uic.edu )

University of Illinois at Chicago

Inna Partin-Vaisband

University of Illinois at Chicago

\section{Research Article}

Keywords: computer-aided design, CAD, electronic design automation, EDA, pathfinding, global routing, machine learning, deep learning, deep neural networks, DeconvNets, convolutional neural networks, ConvNets, generative adversarial networks, GANs, variational autoencoders, VAEs

Posted Date: January 6th, 2022

DOI: https://doi.org/10.21203/rs.3.rs-1194146/v1

License: (1) This work is licensed under a Creative Commons Attribution 4.0 International License. Read Full License 


\title{
Multiterminal Pathfinding in Practical VLSI Systems with Deep Neural Networks
}

\author{
Dmitry Utyamishev ${ }^{1,+, *}$ and Inna Partin-Vaisband ${ }^{2,+}$ \\ ${ }^{1}$ University of Illinois at Chicago, Electrical and Computer Engineering, Chicago, 60607, USA \\ ${ }^{2}$ University of Illinois at Chicago, Electrical and Computer Engineering, Chicago, 60607, USA \\ *dutyam2@uic.edu \\ ${ }^{+}$these authors contributed equally to this work
}

\begin{abstract}
A multiterminal obstacle-avoiding pathfinding approach is proposed. The approach is inspired by deep image learning. The key idea is based on training a conditional generative adversarial network (cGAN) to interpret a pathfinding task as a graphical bitmap and consequently map a pathfinding task onto a pathfinding solution represented by another bitmap. To enable the proposed cGAN pathfinding, a methodology for generating synthetic dataset is also proposed. The cGAN model is implemented in Python/Keras, trained on synthetically generated data, evaluated on practical VLSI benchmarks, and compared with state-of-the-art. Due to effective parallelization on GPU hardware, the proposed approach yields a state-of-the-art like wirelength and a better runtime and throughput for moderately complex pathfinding tasks. However, the runtime and throughput with the proposed approach remain constant with an increasing task complexity, promising orders of magnitude improvement over state-of-the-art in complex pathfinding tasks. The cGAN pathfinder can be exploited in numerous high throughput applications, such as, navigation, tracking, and routing in complex VLSI systems. The last is of particular interest to this work.

Keywords-computer-aided design, CAD, electronic design automation, EDA, pathfinding, global routing, machine learning, deep learning, deep neural networks, DeconvNets, convolutional neural networks, ConvNets, generative adversarial networks, GANs, variational autoencoders, VAEs
\end{abstract}

\section{INTRODUCTION}

Multiterminal pathfinding in presence of obstacles is a task of finding an optimal (e.g., shortest) path between two or more placed terminals. While being computationally hard, this is one of the fundamental navigation problems, required in a wide range of applications, from mobile robot path planning, navigation, wayfinding, and tracking to routing wires in printed circuit boards (PCBs) and integrated circuits (ICs). In this paper, IC global routing will be exploited as a demonstrating vehicle for the proposed pathfinding approach.

Due to the excessively large search space, modern multiterminal pathfindng tasks cannot be optimally solved in reasonable time. To mitigate the computational complexity of pathfinding, existing solvers use approximation heuristics, producing sub-optimal solutions in reasonable time. The traditional deterministic pathfinding approaches typically exhibit the following design flow: a multiterminal pathfinding task is decomposed into multiple two-terminal shortest path tasks and these simpler tasks are solved individually.
Modern pathfinding and routing approaches vary primarily by algorithms for pathfinding decomposition and two-terminal shortest path search as well as by algorithm convergence criteria. The primary limitation of these approaches is the unpredictability of pathfinding convergence and performance. Several important machine learning (ML) approaches have recently been proposed to alleviate the unpredictability issue. Guided by the ML insight, these approaches, however, still rely on poorly parallelizable computational methods, such as the shortest path search. While neural networks (NNs) typically utilize highly parallelizable topologies (i.e., a single instruction can be simultaneously performed with multiple data points on multiple processing units), existing pathfinding algorithms are series in their nature and their runtime cannot be efficiently shortened through parallelization (i.e., instructions are highly inter-dependent and should be executed in order). With the poorly scalable traditional methods and increasing complexity of modern pathfinding applications (e.g., number of terminals and obstacles in modern ICs), runtime and throughput have become a major concern.

In this work, an end-to-end ML pathfinding is proposed. With this approach, ML models are trained on routed multiterminal training samples and exploited to solve unseen test cases. Specifically, the traditional multiterminal obstacleavoiding pathfinding task is mapped onto a modern image manipulation task and solved with a generative $\mathrm{NN}$. It is shown that a properly designed deep $\mathrm{NN}$, trained on robust reference data can efficiently learn and detect routing patterns in inference and determine and execute preferred pathfinding heuristics. As a result, all unseen pathfinding test cases are routed with state-of-the-art wirelength and those complex test cases are routed within a fraction of runtime as compared with existing pathfinders. The significant increase in performance is possible due to the following factors:

1) Reducing the multiterminal pathfinding task to the image-to-image manipulation task: Pathfinding inputs and outputs are mapped onto 2D bitmaps.

2) Accumulating pathfinding information over various tasks, continuously increasing pathfinding performance in new tasks with similar obstacle configuration: This approach is in particular effective in typical IC routing 
use cases, in which thousands of nets are routed within the same placement configuration of standard cells, or autonomous vehicle driving navigation problems, where thousands of autonomous vehicle drives on different routes in the same city or region

3) Efficiently generating a synthetic robust dataset of solved pathfinding task instances: The proposed methodology for generating pathfinding data enables training of a truly deep NN, overcoming a major concern of limited existing multiterminal shortest path data.

4) Parallelizing the pathfinding process for systematic execution on parallel processing hardware accelerators: While pathfinding parallelization is limited with traditional approaches, the parallel nature of ML branchless computation allows to seamlessly and efficiently process pathfinding on GPU, TPU, NPU or other parallel processing hardware in a simultaneous manner and with no overhead.

As a result, the proposed method opens new directions for parallelization of multiterminal pathfinding and scaling the capabilities of its applications, such as of the computeraided IC global routing. The rest of the paper is organized as follows. The background on hardware acceleration based parallelization and ML approaches for image manipulation is provided in Section II. The proposed ML system and design considerations are described in Section III. Evaluation methods and experimental results are presented in Section IV. The paper is concluded in Section V.

\section{BACKGROUND}

In this research, multiterminal obstacle-avoiding task is considered as a rectangular array of labeled tiles. In an input array, each tile is marked as an obstacle, terminal, or empty. During the pathfinding process, some of the empty tiles are marked as path tiles. The primary objective of a pathfinder is determining the tile-to-tile path, connecting all (two or more) terminal tiles. Two terminal tiles are considered connected if there is a set of adjacent (via a shared edge) non-obstacle tiles within the path that includes the two terminal tiles.

The expected output set of path tiles comprises a minimum number of path tiles (i.e., minimum length) while connecting all terminals within the array of tiles and does not intersect with the obstacle tiles set. This task is NP-hard, but can be approximately decomposed and solved in polynomial time with reasonable path length overhead. Existing multiterminal pathfinding solutions are described in subsection A. Machine learning methods exploited in this paper are explained in subsection B.

\section{A. Traditional multiterminal pathfinding}

Traditional multiterminal pathfinding approaches are based on minimum rectilinear Steiner tree (MRST) approximation. With MRST, a multiterminal pathfinding task can be split into multiple terminal-to-terminal pathfinding tasks, using additional auxiliary nodes (i.e., Steiner split-point nodes), and a path between two tiles can be determined with two-point pathfinding algorithm in polynomial time.
Decomposition of a multiterminal pathfinding task into multiple terminal-to-terminal pathfinding tasks is, however, also NP-hard. Thus, approximating methods such as minimal spanning tree (MST) are often utilized instead of MRST, yielding suboptimal, yet computationally preferred solutions with a typical computational complexity of $\left.O\left(n \cdot \log (n)^{2}\right)\right)$, where $n$ is the overall number of tiles [1], [2]. A primary advantage of the MST method is that the total length of the generated path is within certain bounds of the optimal length. Another method for mitigating the MRST complexity is lookup tables [3], [4]. These methods typically exhibit polynomial time complexity, trading off the optimal length for a shorter execution runtime.

Once the original multiterminal pathfinding task is successfully split into multiple two-terminal sub-tasks, best-first search algorithms are commonly utilized for determining paths between the original terminals and split-point nodes, as well as paths between different split-points of the Steiner tree. Methods such as pattern routing, negotiated-congestion routing, and integer linear programming (ILP) are utilized in modern global routers to speed those easy-to-route nets up and resolve difficult-to-route regions. An optimal MRST and sub-optimal MST routing is illustrated in Figure 1.

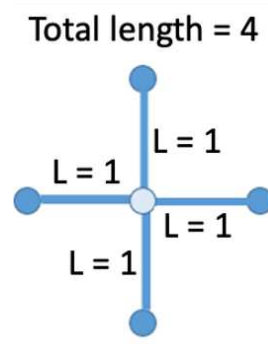

(a)

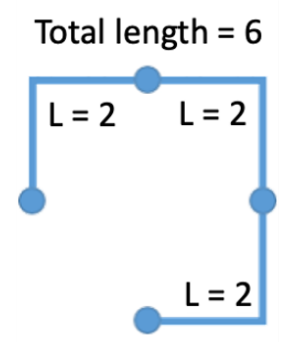

(b)
Fig. 1: Pathfinding of a four-terminal input with (a) the optimal terminal-to-split path (as determined by the MRST approach), resulting in the shortest total path length, and (b) terminalto-terminal path (as determined by the MST approximation), yielding suboptimal (i.e., longer than in (a)) total path length.

Albeit the progress in IC routing, at the core of the existing pathfinding solutions remains the reliance on graph traversing and similar algorithms that yield non regular and, thus, poorly parallelizable computational methods. While neural networks typically utilize highly parallelizable topologies (i.e., a single instruction can simultaneously be performed with multiple data points on multiple processing units), existing routers are series in their nature and their runtime cannot be efficiently shortened through parallelization (i.e., instructions are highly interdependent and should be executed in order). Such pathfinding with poorly scalable traditional methods becomes even more challenging with the increasing number of terminals and obstacles in modern VLSI systems.

\section{B. Existing ML-based multiterminal pathfinding}

While ML driven pathfinding is of particular interest, most of the existing pathfinding approaches utilize ML to predict 
convergence, wirelength, and other path characteristics with a certain initialization state [5]-[14]. Alternatively, several important attempts to route paths with a NN exhibit limited scalability and training restrictions. For example, a generative deep learning model utilized in [15] for IC pathfinding exhibits limited maximum resolution (up to $64 \times 64$ pathfinding tiles) and cannot replace traditional pathfinders in practical applications. Performance of the variational autoencoder (VAE) architecture used in [15] is known to significantly decrease with an increasing input resolution. Furthermore, the performance of this supervised method is a strong function of the robustness and quality of the training set, yielding another primary concern. In order to produce a robust prediction model, supervised ML methods require significant amount of training data (i.e., pathfinding task-solution pairs). While actual IC physical design data is proprietary and not available in required amount, and straight-forward method of generating random inputs and solving them with traditional pathfinding algorithms is not feasible due to computational complexity of pathfinding. Therefore, a novel method to generate synthetic training data for supervised ML pathfinding is required.

Another VAE-based model [16] also exploits the image representation of a two-dimensional IC to route paths in analog ICs. In this work, the labeled data for the supervised training comprises a limited set of existing routed analog ICs, as hand-solved by the human experts. To obtain reasonable performance with such a limited training set, ICs has been aggressively downsampled down to $64 \times 64$ tiles (similar to [15]). While the model performs well in analog domain, the limitations of the training set and ML architecture make these approaches impractical for pathfinding in modern digital ICs and other applications.

Additional recent works explore ML pathfinding solutions within a simplified design space (e.g., small input space, no obstacles, two-pin instances) [17], [18]. The importance of a large robust training set to enable deep learning is emphasized in [17] and a methodology for generating a set of multiterminal obstacle-avoiding pathfinding tasks is proposed in the same paper. However, how to route numerous generated tasks with a state-of-the-art like path length is still an open question. To address this question a methodology is proposed in this paper for efficiently generating a large amount of practical training data.

A breakthrough in ML physical design has recently been achieved by researchers from Google who developed a deep learning approach for IC floorplanning [19]. In this work, the whole IC gate graph is embedded into a low-resolution latent space and upsampled with a stack of convolutional layers to a two-dimensional image representation of the placed IC. Graphembedding models are used to represent a preferred placement graph [20]. Based on the reported results, months of traditional floorplanning are reduced to a few hours with [19]. While the floorplanning solution cannot be seamlessly adapted for global routing and multiterminal pathfinding, this work is an important step toward a physical design end-to-end learning in modern ICs. The goal of this paper is to initiate a similar paradigm shift in multiterminal pathfinding.

\section{Pathfinding as bitmap translation}

Modern ML image processing solutions are reduced to convolution operations (e.g., within a convolutional kernel or convolutional layers of deep neural networks), that are decomposed into a large number of small independent matrix multiplications. Hardware accelerators can, therefore, be efficiently utilized in this type of computation with large number of cores and parallel access to local and shared memory. Thus, mapping an array of IC tiles onto a 2D image transforms an inherently sequential task to a naturally parallelizable one, enabling efficient utilization of hardware acceleration platforms. Identifying an appropriate class of imaging problems and effectively representing pathfinding tasks within that class is, therefore, a primary objective. Yet another objective is to design a large training dataset of robust pathfinding tasks (2D arrays with varying number and location of terminals and obstacles) and corresponding solutions (arrays with state-ofthe-art like paths), as required for typical ML imaging training.

Imaging translation is a class of problems which focuses on learning the per-pixel mapping from an input bitmap to an output bitmap, hence translating one possible data representation into another. This approach is useful in various domains and applications, such as style transfer, inpainting, and object transfiguration and typically exploited for transforming and repairing photos. Similarly, we propose to reconsider a pathfinding task as a problem of image reconstruction in which the path tiles are the missing image parts which are reconstructed using image translation. Existing image translation solutions are typically based on generative NNs and thus, highly parallelizable. A generative $\mathrm{NN}$ is designed in this paper to demonstrate pathfinding with image translation, yielding a fundamentally novel, highly scalable and parallelizable solution for the multiterminal pathfinding task.

\section{Generative machine learning}

$\mathrm{ML}$ is a set of computational techniques that can be exploited for searching complex patterns in large volume of data and predicting the output based on provided input and ML parameters. Supervised learning is one type of ML paradigm, that utilizes training data for determining ML model parameters. Each training sample comprises an input and the corresponding true label. During the training, ML model is iteratively updated to minimize the error between its output and the true label.

VAEs have been demonstrated as a powerful solution for image-to-image processing problems, such as image colorization, stylization, or inpainting. A VAE is a deep NN (DNN) that combines a recognition and a generative models. The recognition model (commonly designed as a convolutional neural network (ConvNet)) encodes DNN input into a vector of latent state probabilistic distributions of learned attributes, while the generative model (commonly designed as a deconvolutional neural network (DeconvNet)) decodes the randomly sampled latent state distributions into the DNN output.

Convolutional neural networks have been proven as a preferred ML architecture for efficient detection of complex local patterns in 2D maps. A ConvNet is a stack of convolutional 
and pooling layers. Each convolutional layer is defined by a convolutional kernel that slides over the inputs of the layer, generating a local map based on the local layer features. Two important hyperparameters of a convolutional layer are stride and padding. While stride controls the sliding of the kernel over the input volume, padding maintains the dimensionality of the data. The objective of a pooling layer is to reduce the dimensionality of data, abstracting the information about complex features as this information propagates forward through a ConvNet. The inner (i.e., with the lowest dimension) latent space represents attributes of a given input as a probability distribution. When decoding from the latent space, latent attributes are sampled from corresponding distributions to generate a vector, that is further processed with deconvolutional layers. The concepts of kernel size, stride, padding, and pooling are exemplified based on a $4 \times 4$ bitmap with a $3 \times 3$ kernel, stride of one, and pooling that prioritizes maximum values, as shown in Figure 2.

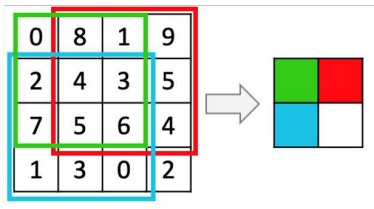

(a)

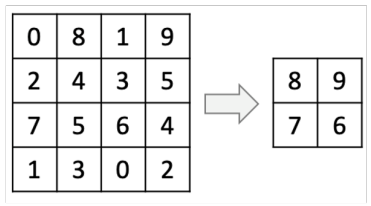

(b)

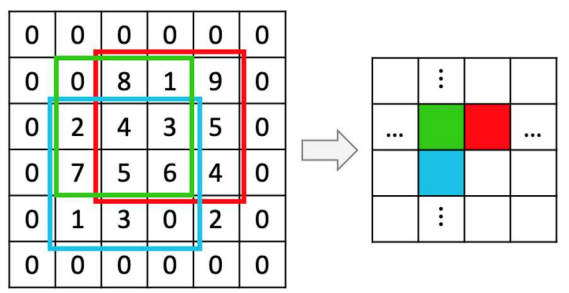

(c)

Fig. 2: Illustration of ConvNet hyperparameters on a $4 \times 4$ bitmap with a $3 \times 3$ kernel, (a) a convolutional $3 \times 3$ kernel with stride of one reduces the bitmap dimension from $4 \times 4$ to $2 \times 2$, (b) dimensionality is reduced with max-pooling, and (c) bitmap dimension is maintained from (a) to (b) with intermediate zero padding.

Deconvolutional neural networks are commonly used to decode a low dimensional data space into a dimensionally higher space. The DeconvNet topology is similar to ConvNet, except for the upsampling DeconvNet layers that replace the pooling ConvNet layers.

Conditional generative adversarial networks (cGANs) are an advanced ML training approach. With this approach, generator (VAE is commonly used as a cGAN generator) and discriminator submodels are utilized and conditioned by a certain input (e.g., a generated path is conditioned by certain placed terminals and obstacles). A discriminator convolutional model is trained to classify an output bitmaps as a true label or ML generated bitmap. Simultaneously, the generator is trained to produce output bitmaps that cannot be recognized by the discriminator as ML generated. As a result, the error between the generated and expected (i.e., true label) output bitmaps is reduced over successive training iterations. The adversarial nature of the architecture allows the generator submodel to simultaneously learn the mapping between 1) the input (e.g., pathfinding array with marked obstacles and terminals) and generated output (e.g., the corresponding routed path) bitmaps, and 2) the true label (e.g., a path from the training dataset) and generated output. As opposed to conventional dictionary based approaches and lookup tables, a cGAN is not limited to reproducing known outputs by key, but can generate routed paths from previously unseen inputs. The training process of cGAN ML model is illustrated in Figure 3.

Performance of ML system is a strong function of a training set and training time. Model convergence time increases with the increasing number of training data samples. Alternatively, as the number of training samples is reduced, or the diversity of training data becomes limited, the risk of model overfitting is increased, yielding high performance with a training set but low performance with unseen input data. Typical imaging training sets comprise up to a few thousands data samples. In the next section, the formulation of a multiterminal pathfinding as an imaging task is proposed and ML system design considerations are described. Design solutions that facilitate generation of a robust training set and convergence of the model in reasonable time without overfitting are also proposed.

\section{PROPOSED PATHFINDING SYSTEM}

The proposed workflow of ML-based pathfinder comprises three key phases. A training set of 2D bitmaps (i.e., routed and unrouted bitmap pairs) is generated during the first phase (see subsection A). During the second phase, a cGAN model is trained on the training set with physics aware loss function (see subsection B). While the generation of training set and the training are time consuming tasks (e.g., can take hundreds of hours on NVIDIA GTX1080 platform), these tasks are not necessarily performed from scratch. Existing training sets from other ML pathfinding and routing systems can be reused and enhanced. Finally, transfer learning and learning with partial layout information (e.g., information about certain standard cells, as shown in Section 4) can be utilized to fine-tune pre-trained models, enhancing the overall pathfinding performance. The process of training set generation and training itself is, therefore, expected to improve over generations of VLSI systems.

During the third (inference) phase, pathfinding input data is parsed and mapped into a pathfinding solution with a properly trained generative ML model. A typical concern with the proposed approach is the connectivity of a generated path. While in imaging problems, a missing or incorrect pixel has little effect on image perception, a routing path with a missing pixel exhibits an open circuit and thus invalid. To maintain path connectivity, those paths that are generated with disconnected clusters (based on experimental results, less than ten) are postprocessed.

Each of the framework phases is explained in details in the following subsections. The methodology to efficiently generate a robust training set is described in subsection A. The architecture of the cGAN pathfinder and the proposed physics aware loss function are explained in subsection $\mathrm{B}$. The 


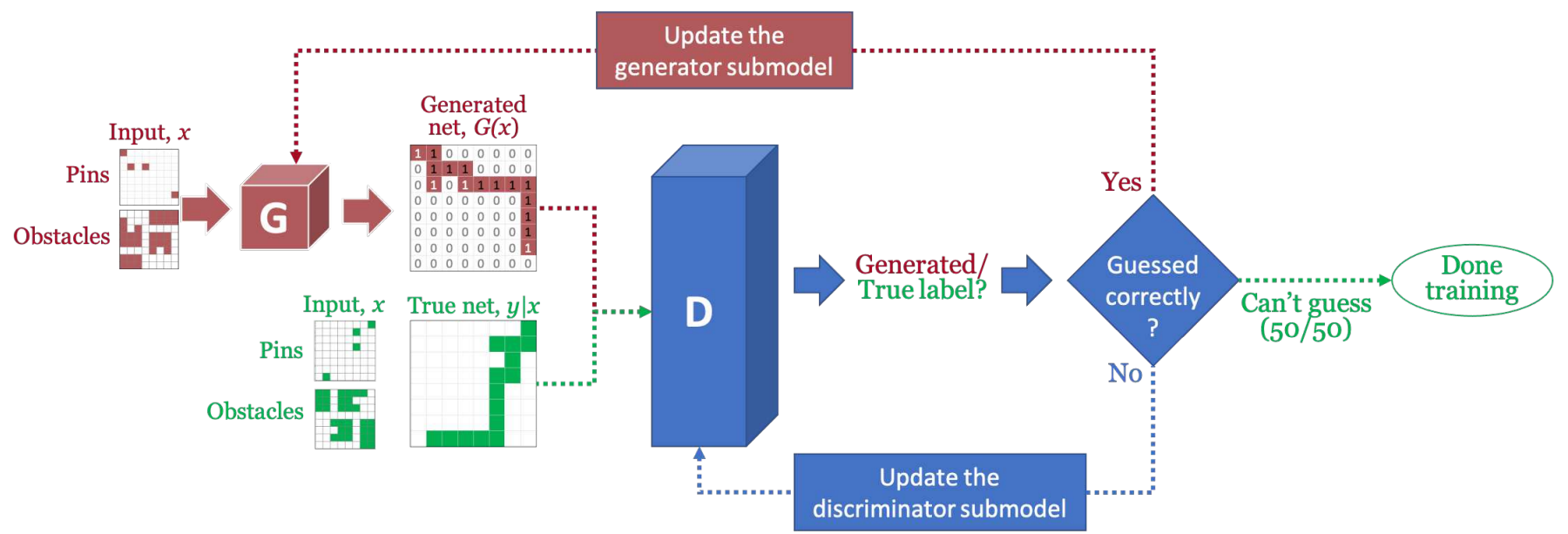

Fig. 3: Training a cGAN to generate robust multiterminal paths.

postprocessing algorithm for merging clustered cGAN paths is presented in subsection $\mathrm{C}$.

\section{A. Training set generation}

To approach a pathfinding challenge as a supervised ML task, the ML model needs to be trained on a set of pathfinding reference samples (i.e., bitmaps with terminals, obstacles, and corresponding paths). The pathfinding reference samples are synthetically generated in this paper.

The key idea is merging several low resolution, reference samples into more complex samples. Note that pathfinding optimality in the high resolution training samples is not required for efficient ML training. Alternatively, the training samples should capture a broad variety of pathfinding patterns. First, a large set of pathfinding tasks is generated within small (i.e., $8 \times 8$ to $128 \times 128$ ) rectangular bitmaps. Each small bitmap comprises at least one terminal or a path segment placed on the bitmap perimeter (i.e., edge terminal or edge path segment). These small bitmaps are optimally solved with exhaustive pathfinding methods. A valid merge of solved paths into a longer, more complex path within a larger bitmap is accomplished by joining two bitmaps via two edge segments. For that purpose, smaller bitmaps can be rotated and flipped as needed. The merging process continues until the resulted bitmap reaches the target sample resolution. Examples of valid and non-valid merges are shown in Figure 4.

The flow diagram of the training set generation is shown in Figure 5. At each iteration, a bitmap is randomly selected from a pool of bitmaps and matched with another random bitmap from the pool for a valid merging. Note that bitmaps generated in this manner tend to exhibit statistically significant difference in path density at the edge tiles, increasing the risk of model overfitting during training. To mitigate overfitting, each path resulted from a valid merging is shifted in a random direction, as shown in Figure 4. The grouped and shifted bitmap is added to the pool and the process continues to the next iteration. A model trained on the resulted training set is expected to capture broad pathfinding rules in presence of obstacles. To capture a system specific obstacle constraints, another fine-tuned data set is generated. Samples in this set are generated in the following manner. Tiles from a typical layout are randomly sampled and combined into a small bitmap (e.g., $128 \times 128$ ). The bitmap is utilized to generate thousands of pathfinding tasks with numerous randomly placed terminals, which are routed with conventional methods. Based on experimental results, including the fine-tuned data within the training set significantly increases the saturation speed of the trained model.

\section{B. Neural network architecture}

The typical conditional adversarial loss is defined as

$$
\begin{aligned}
L_{c G A N}= & \mathbb{E}_{x, y}[\log D(y \mid x)]+ \\
& \mathbb{E}_{x, z}[\log (1-D(G(x, z) \mid x))],
\end{aligned}
$$

where $x$ is the input bitmap, $y$ is the expected (routed) output bitmap (i.e., the true label), $z$ is the random noise, generator $G: x, z \rightarrow \hat{y}$ aims at minimizing the loss, and the adversarial discriminator $D: x, y \rightarrow\{$ 'true','generated' $\}$ aims to maximize the loss.

While in traditional cGANs, random noise is utilized to generate different stochastic outputs, in a typical pathfinder, the preferred output is not random but determined based on physical IC characteristics. In this paper, the cGAN is designed without the random noise but enhanced with physics-aware path generation reconstruction loss function, $L_{r}$. The trained generator $G^{*}$ is, therefore, determined by

$$
G^{*}=\arg \min _{G} \max _{D} L_{c G A N}(G, D)+\lambda L_{r}(G) .
$$

To understand how the $L_{r}(G)$ is determined, consider the following definitions for formulating the pathfinding task as a supervised ML task. Let $X$ and $Y$ be the sets of, respectively, unrouted bitmaps with placed terminals and obstacles and corresponding single-path routed bitmaps. A pathfinding task is to find the preferred pathfinding path of tiles, $y_{x} \in Y$, connecting a certain number of placed terminals under certain obstacle constraints, as defined by $x \in X$. For an unrouted $n \times n$ bitmap $x \in X$, the corresponding single-path routed bitmap $y_{x} \in Y$ is an $n \times n$ bitmap of tiles $(i, j), 1 \leq i, j \leq n$, 
Optimally routed compact bitmaps

$\mathrm{b}_{1}$

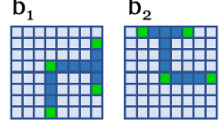

$\mathrm{b}_{3}$
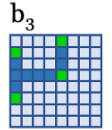

$\mathrm{b}_{5}$

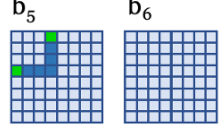

(a) $\mathrm{b}_{4}$
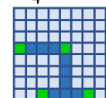

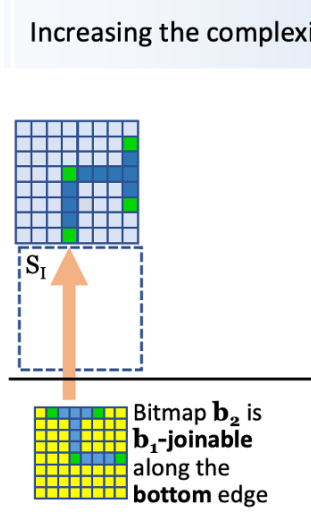

(b)

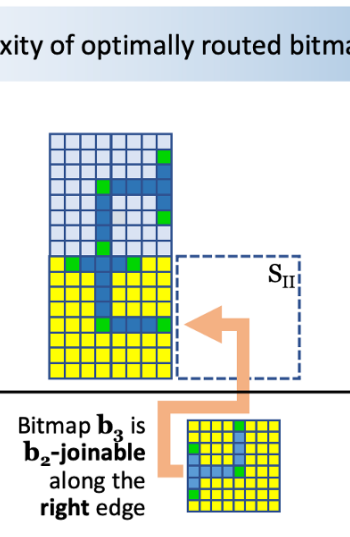

(c)

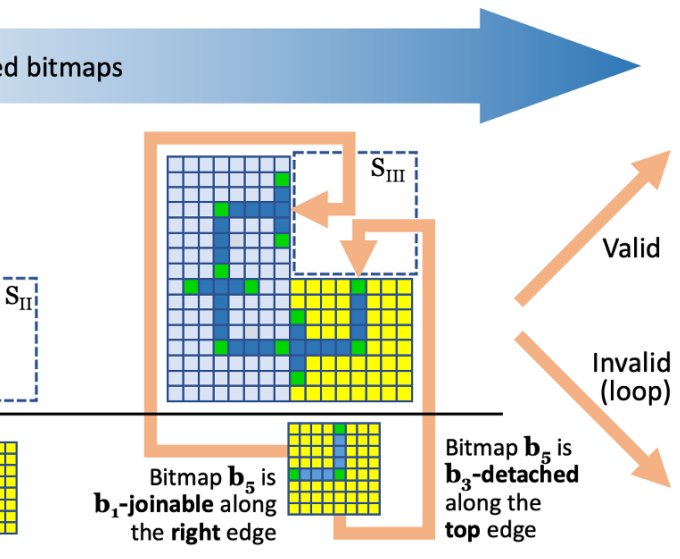

(d)
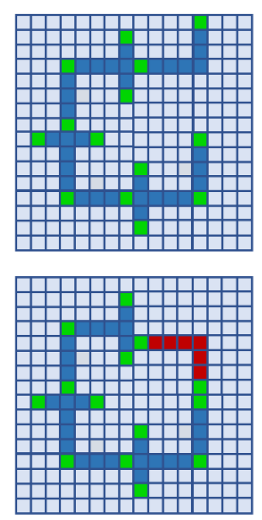

(e)

Fig. 4: Illustration of the proposed flow for generating a training sample of a robust routed path. (a) A set of low-resolution optimally routed bitmaps. (b) Bitmap b1 is randomly selected and placed. Given the single bitmap, b1, adjacent to the unprocessed space SI, the bitmap, b2, is randomly selected from the list of b1-joinable bitmaps and placed in SI along the bottom edge of b1. (c) Given the single bitmap, b2, adjacent to the unprocessed space SII, the bitmap, b3, is randomly selected from the list of b2-joinable bitmaps and placed in SII along the right edge of b2. (d) Given the two bitmaps, b1 and b3, adjacent to the unprocessed space SIII, the bitmap, b5, is randomly selected from the intersection of the b1-joinable and b3-detached bitmaps and placed in SIII along the right edge of b1 and top edge of b3. (e) As a result, a robust routed valid bitmap with $4 \mathrm{x}$ resolution is generated (top). Alternatively, selecting the last bitmap from the intersection of the b1- and b3-joinable bitmaps results in an invalid circular path (bottom). Thus, bitmaps are always selected from the intersection of a joinable and detached lists, as shown in the flowchart in Figure 5.

where each tile is associated with a binary score, $y(i, j)=0$ or $y(i, j)=1$ if the tile $(i, j)$ is, respectively, excluded from or included within the preferred output path. These definitions are illustrated in Figure 6.

Note that the overall objective is to maximize the total number of routed paths, while minimizing the total length of the pathfinding solution. In the ML domain, the goal is to train a ML system $\hat{Y}=\left\lfloor f\left(X, G^{*}\right)+0.5\right\rfloor$ that for each $x \in X$ provides the conditional probability of each tile, $\hat{y}_{x}(i, j)$, to be either included within (i.e., $\left.f_{(i, j)} \geq 0.5\right)$ or excluded from (i.e., $\left.f_{(i, j)}<0.5\right)$ the preferred pathfinding solution,

$$
f_{(i, j)}\left(X, G^{*}\right)=P_{G^{*}}\left(\hat{y}_{x(i, j)}=1 \mid x\right),
$$

where $G^{*}$ is the generator model trained based on the conditional probability distribution of the input features, $x_{i}$, and output observations, $y_{i}$ (i.e., true labels), as defined by (2). The training data set $\left\{\left(x_{k}, y_{k}\right)\right\}_{k=1}^{N}$ comprises $N$ synthetic pathfinding tasks in the bitmap representation and $N$ corresponding reference single-path routed bitmaps (i.e., the true labels).

Mean square error (MSE) loss function is typically used with autoencoders for evaluating sum of squared distances between the predicted values and true labels [21], [22]. For the pathfinding task, MSE counts the number of tiles that marked differently (' 0 ' vs ' 1 ') with the true label and generated solution. Note that for an $n \times n$ optimally routed bitmap, the number of empty $\left(f_{(i, j)}<0.5\right)$ and routed $\left(f_{(i, j)}>=0.5\right)$ tiles scales as, respectively, $\mathcal{O}\left(n^{2}\right)$ and $\mathcal{O}(n)$ with $n$ ). This unbalanced nature of the pathfinding data set fosters prioritization of the "all zeros" solution (i.e., an empty layout), which validity further increases with the increasing bitmap size $n$.
Thus, MSE loss function is impractical for ML pathfinding.

To account for specifics of path minimization task, a custom loss function is proposed. The custom loss function is designed to penalize the model if the number of tiles, $\hat{n}_{t}$, included by the model within an output path is different from the number of tiles in a reference output path, $n_{t}$. The penalties for $\hat{n}_{t}$ exceeding and falling short of $n_{t}$ differ. A path with redundant tiles is not optimal in terms of the path length, but is legal if it connects all the input terminals. Alternatively, if $\hat{n}_{t}<n_{t}$ and the reference path is optimal, then some components in the model solution are disconnected and the path is, therefore, incorrect. In particular, the $\hat{n}_{t}<n_{t}$ penalization pertain to the "all-zeros" local minimum. Given a predicted routed bitmap, $\hat{y}$, and a reference bitmap, $y$, the proposed loss function accounts for $\left|\hat{n}_{t}-n_{t}\right| \neq 0$ with penalty rate of $k_{\text {sub-opt }}$ and for incomplete paths with additional penalty rate of $k_{e r r}$, yielding

$$
L_{r}=\operatorname{MSE}(\hat{y}, y) \cdot\left(1+k_{\text {sub-opt }} \cdot \text { step } \cdot \text { distance }\right),
$$

where

$$
\begin{gathered}
\text { distance }=\sum_{i, j} H\left(\hat{y}_{i, j}\right)-\sum_{i, j} H\left(y_{i, j}\right) \\
\text { step }=k_{\text {err }} \cdot \operatorname{sign}(\text { distance }-1)+1 .
\end{gathered}
$$

Here $H(\cdot)$ is the Heaviside step function. In this paper, the proposed loss function is used with $k_{\text {sub-opt }}=10^{-3}$ and $k_{\text {err }}=$ $10^{2}$.

The proposed generator is designed as a multi-stage NN. All tiles with the individual obstacle and terminal indicators are fed as ML features into the input channels of the generator. The input dimension of the network is therefore $2 n^{2}$, as determined by the total number of features of the $n \times n$ tile bitmap. 

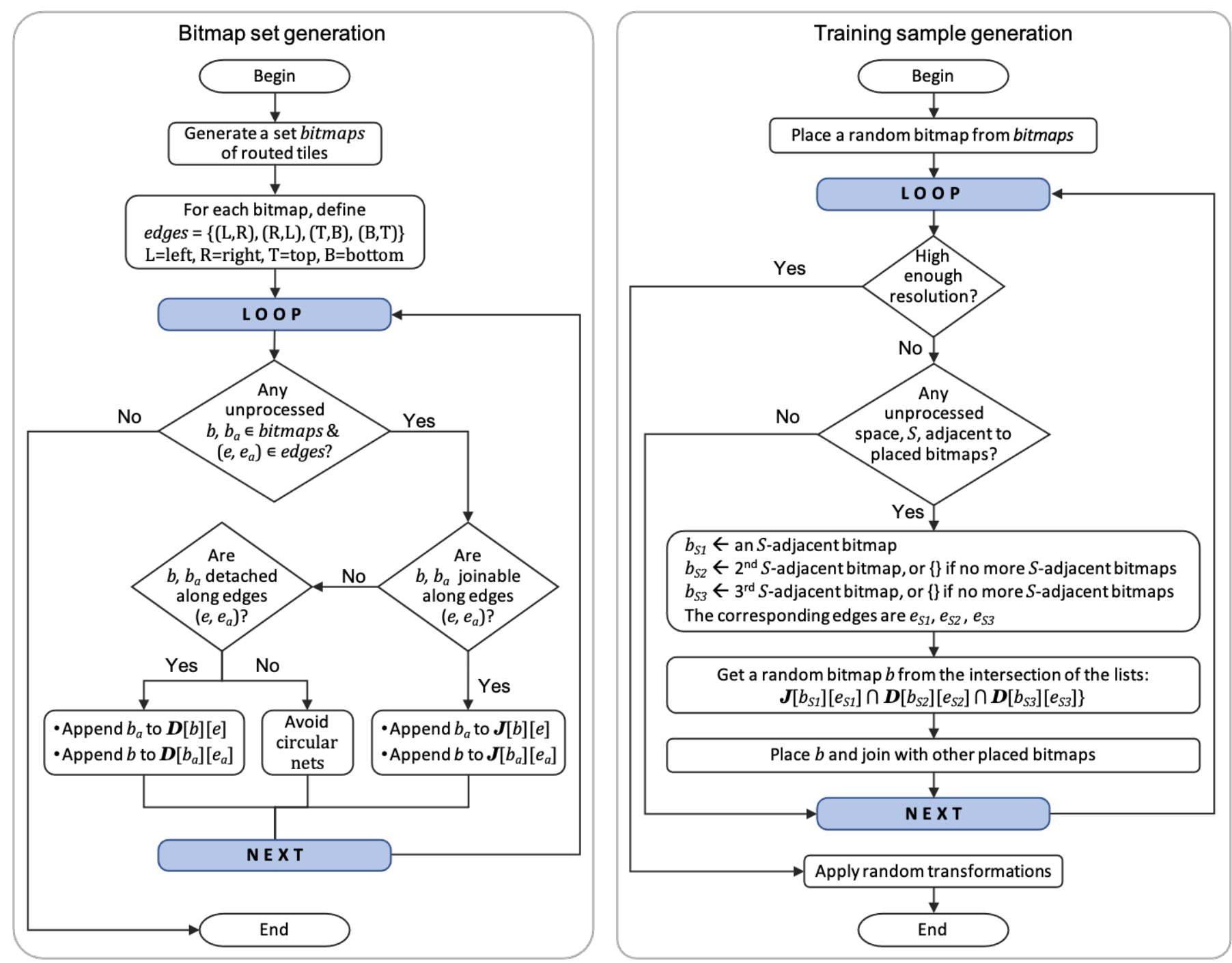

Fig. 5: Flow diagram of the training set generation algorithm. On the left is the flow for generating small bitmaps. These bitmaps are optimally routed with optimal algorithm. On the right is the flow for generating complex training dataset by joining the routed small bitmaps.

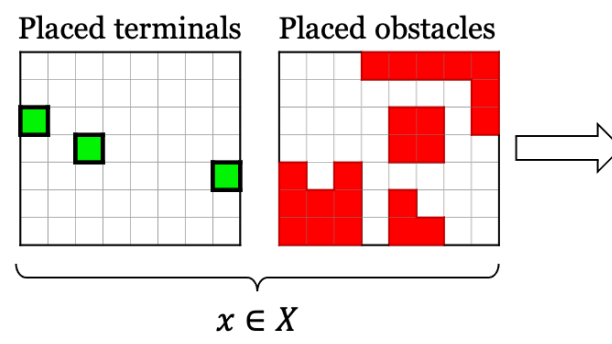

Fig. 6: Illustration of a single pathfinding task with input bitmap $x \in X$ and the corresponding solved path $y_{x} \in Y$.

To mitigate the high input dimensionality of the system, a ConvNet based VAE is used. A typical VAE architecture (see Figure 7) is utilized, comprising seven encoding layers, three latent dense layers, seven decoding layers, and a single refining layer. In dense layers, $25 \%$ of inputs is dropped out (i.e., set to zero) at each update during the training to prevent overfitting. The encoder converts the $2 n^{2}$-dimensional input data into an intermediate low-dimensional (i.e., $256 \times 2$ ) data space, using a stack of convolutional layers. The decoder then deconvolves the abstracted data into the $n^{2}$-dimensional routed output space, using a stack of deconvolutional layers. Each of the output values indicates the probability of the corresponding tile to be included within the output path. The final decision to include a tile within the pathfinding output is made based on the decision threshold of 0.5 . If a tile output value exceeds this threshold, the tile is considered to be part of the output path. Unlike other generative models which require more complex training approaches, the proposed $\mathrm{NN}$ configuration is a linear stack of layers and naturally supports error backpropagation throughout the overall network. As a result, efficient training of the VAE generator within the intermediate low-dimensional dense layers is possible. Owing to the stochastic nature of the ML model, new (unseen) pathfinding solutions can be generated based on the training data points (i.e., existing or synthetically generated routed bitmaps) by sampling the intermediate probabilistic space. For optimization reasons, the generator NN is augmented with skip connections between corresponding convolutional and deconvolutional layers, similar to the U-Net [23] NN architecture. 


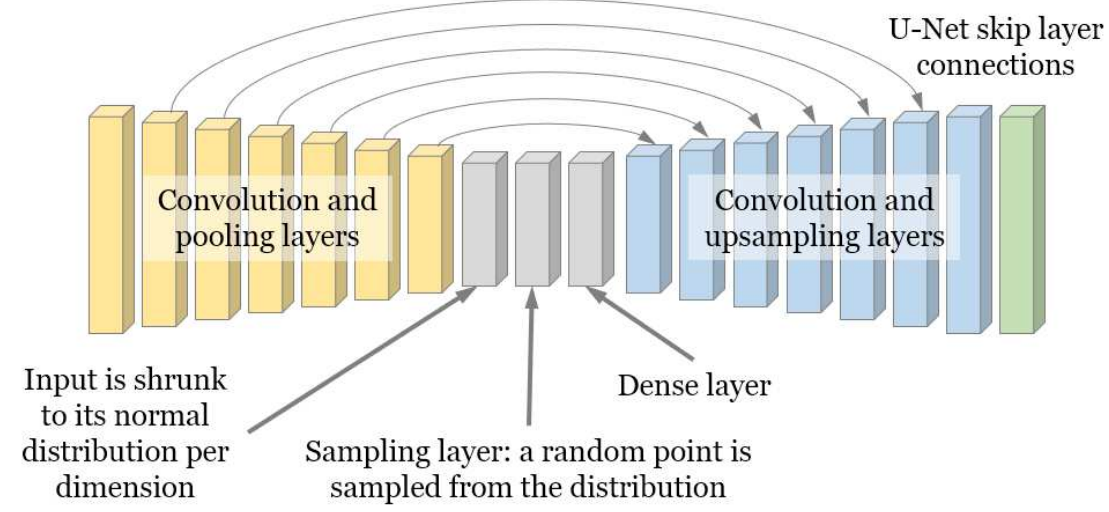

(a)

\begin{tabular}{|c|c|c|c|c|c|c|}
\hline Layer id & Layer type & Number of filters/neurons & Kernel size & Stride & Padding & Activation function \\
\hline 1 & Convolutional & 32 & $3 \times 3$ & 1 & Same & ReLu \\
\hline 2 & Pool & & $2 \times 2$ & & & \\
\hline 3 & Convolutional & 64 & $3 \times 3$ & 1 & Same & ReLu \\
\hline 4 & Pool & & $2 \times 2$ & & & \\
\hline 5 & Convolutional & 128 & $3 \times 3$ & 1 & Same & ReLu \\
\hline 6 & Pool & & $2 \times 2$ & & & \\
\hline 7 & Convolutional & 256 & & & Same & ReLu \\
\hline 8 & Dense & $512^{\circ} 25 \%$ dropout & & & & ReLu \\
\hline 9 & Sampling & & & & & Relu \\
\hline 10 & Dense & $256^{\circ} 25 \%$ dropout & & & & ReLu \\
\hline 11 & Convolutional & 256 & $3 \times 3$ & 1 & Same & ReLu \\
\hline 12 & Upsampling & & $2 \times 2$ & & & \\
\hline 13 & Convolutional & 128 & $3 \times 3$ & 1 & Same & ReLu \\
\hline 14 & Upsampling & & $2 \times 2$ & & & \\
\hline 15 & Convolutional & 64 & $3 \times 3$ & 1 & Same & ReLu \\
\hline 16 & Upsampling & & $2 \times 2$ & & & \\
\hline 17 & Convolutional & 32 & & 1 & Same & Sigmoid \\
\hline
\end{tabular}

(b)

Fig. 7: Architecture of the proposed generator network, (a) block level schematics comprising convolutional, dense, and deconvolutional layers, as well as an example of input features and output, and (b) NN parameters for each layer of the $1024 \times 1024$ pathfinder.

\section{Postprocessing algorithm}

While several missing or corrupted pixels typically go unnoticed in ML generated images, missing path pixels yield an invalid path. A postprocessing algorithm is proposed to merge the few disconnected cGAN output clusters, as needed. As part of the algorithm, an invalid path is processed with the median filter for image noise reduction and the path tiles clusters are connected with the cluster merging algorithm (see Figure 8).

With the proposed algorithm, pairs of closest endpoints (from two different clusters) are identified for all disconnect endpoints based on Manhattan distance. To merge two clusters, the identified closest endpoints are connected with mazerouting algorithm. While the proposed greedy algorithm is generally suboptimal, it has been shown to exhibit optimal results when used to connect only few clusters in cGAN generated paths.

An example of the postprocessed output is shown in Figure 9 . In this case, the cGAN routed path exhibits three disjoint clusters that are joined with the proposed postprocessing algorithm. Note that with a completely random input (i.e., randomly generated number and location of pins and obstacles), most of the unprocessed cGAN routed paths yield between two

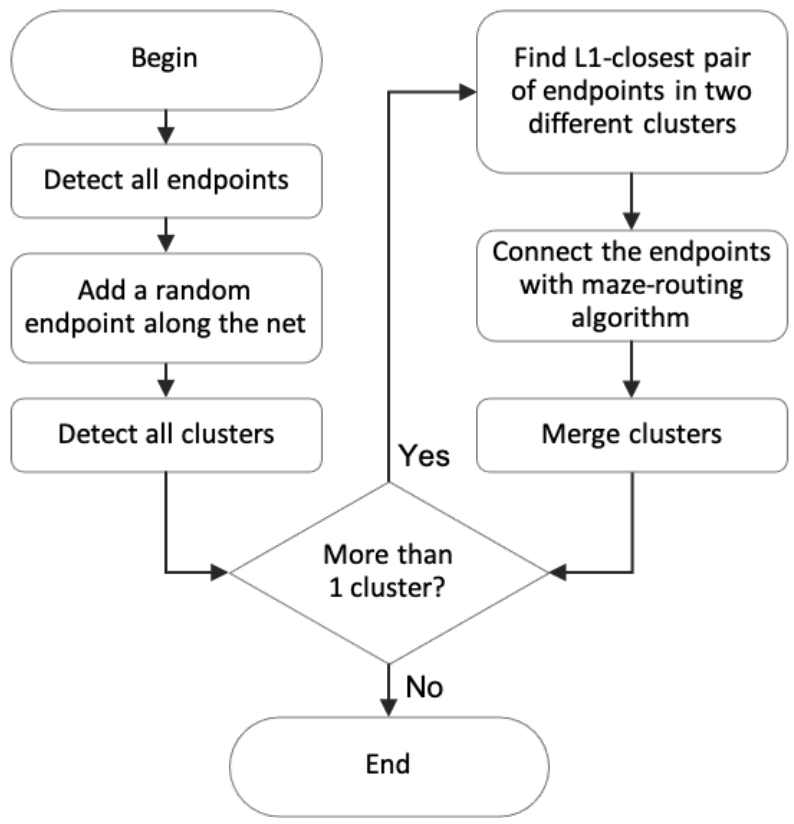

Fig. 8: Flow diagram of the proposed postprocessing method.

and ten disjoint clusters. This behavior is primarily caused by overfitting issues, and can be solved with either the proposed 

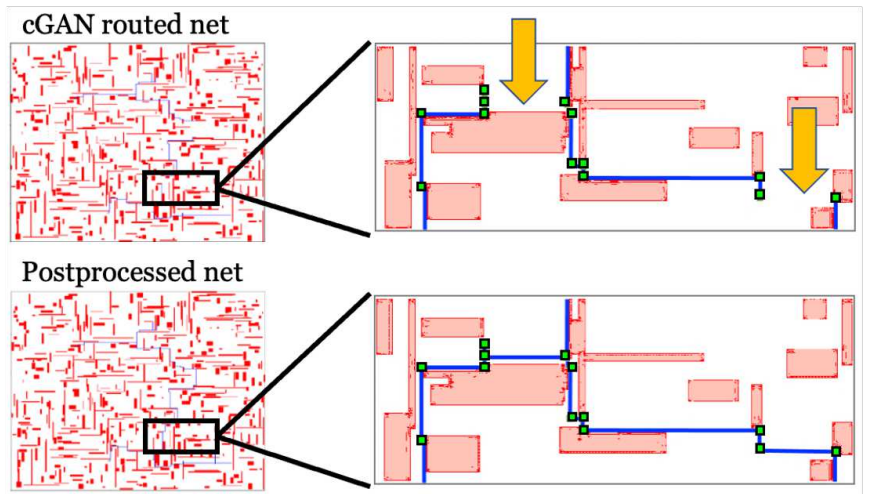

Fig. 9: Raw cGAN output of RT01 and a refined solution. Three disjoint clusters are connected during the postprocessing stage.

postprocessing or fine tuning of training set. When the cGAN model struggles to converge to a correct output, a grid of dots with tile-like size is produced, as shown in Figure 10. This is, however, not a fundamental limitation of the proposed approach, but a constraint of the utilized synthetic training set. With a more heterogeneous training set, that contains larger diversity of generated tile sizes, postprocessing may not be required. Such training set generation methods should be considered in the future.

\section{EVALUATION AND EXPERIMENTAL RESULTS}

The proposed cGAN pathfinder has been tested with the following pathfinding inputs.

1) A set of unseen pathfinding tasks synthetically generated with the proposed algorithm (see subsection III.A.).

2) A set of pathfinding tasks generated by randomly placing rectangular obstacles and terminals within a bitmap. The number and size of the obstacles as well as the number of terminals are all randomly determined.

3) Multiterminal pathfinding benchmarks RT 01-05 [24].

For evaluation purposes, the input data is represented as a $1024 \times 1024 \times 2$ array, in which the first and second channel are the per-tile obstacle and terminal indicators, respectively. Bitmaps smaller than $1024 \times 1024$ are upsized to $1024 \times 1024$ and/or filled with obstacle indicators along the bitmap edge.

\section{A. Evaluation metrics}

The cGAN pathfinding algorithm is evaluated with respect to four primary metrics: correctness of paths (i.e., a correct path must connect all the terminals in a continuous manner), wirelength (as determined by the number of path tiles), runtime, and throughput. To evaluate the correctness of the ML pathfinding, best-find search is utilized to find all connected tiles within the routed path (i.e., those tiles with $f_{(i, j)} \geq 0.5$ ). Each search starts at one of the terminals and progressively constructs a set of visited tiles. At each iteration, the tiles adjacent to the already traversed tiles are added to the set. The search stops when all the tiles have been traversed.

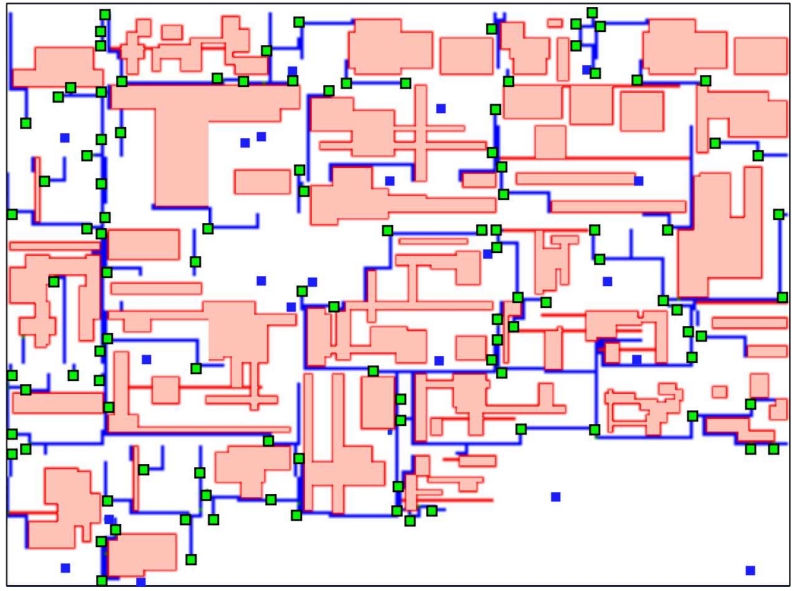

(a)

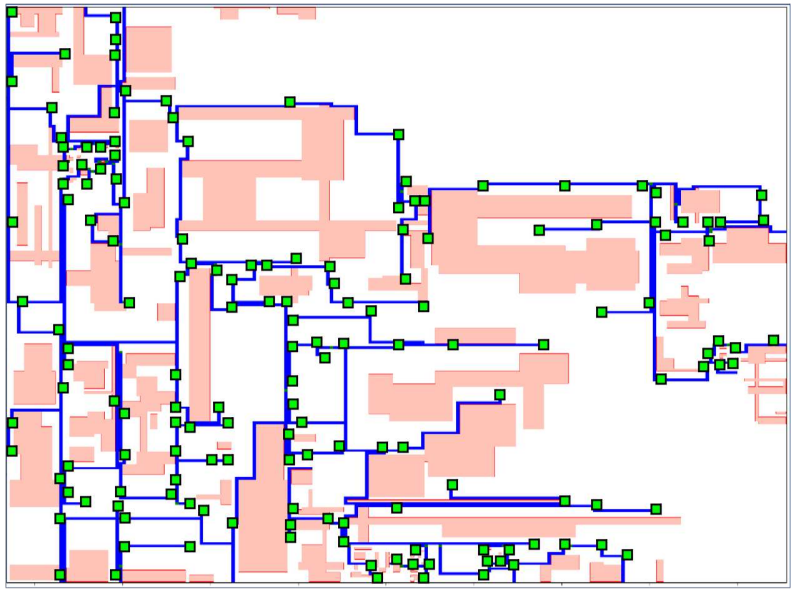

(b)

Fig. 10: Illustration of the model output. Terminals (enlarged for visibility), obstacles, and routed paths are shown in, respectively, green, red, and blue shades. (a) An incorrect output with grid-like path is generated due to the tiled nature of the training set, and (b) A correct output with no scattered path pieces.

\section{B. Experimental results}

The cGAN pathfinder is trained on a synthetically generated pathfinding dataset and tested on both the RT multiterminal pathfinding benchmarks [24] and synthetically and random generated test cases. The tested tasks are not part of the training set and have never been seen by the cGAN model. The cGAN pathfinder is able to generate a path with similar to state-of-the-art length for inputs of different complexity. An example of the cGAN pathfinder output at various stages of training is shown in Figure 11. Performance comparison between the cGAN and state-of-the-art deterministic pathfinding algorithms (ML-OARSMT [24] and FOARS [4]), is listed in the table below for the RT benchmarks. Both deterministic algorithms are based on the look-up table-accelerated decomposition of multiterminal pathfinding. FOARS provides an enhanced method of obstacle-aware decomposition, yielding state-of-the-art performance. While ML-OARSMT yields less competitive performance, it utilizes an open source fundamen- 


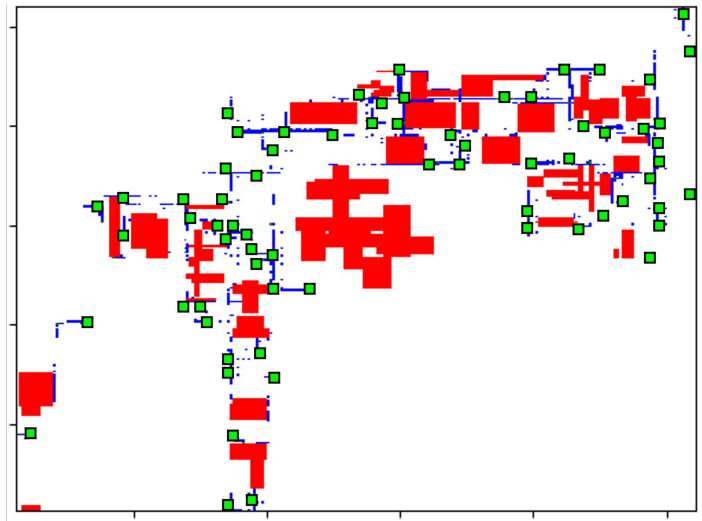

(a)

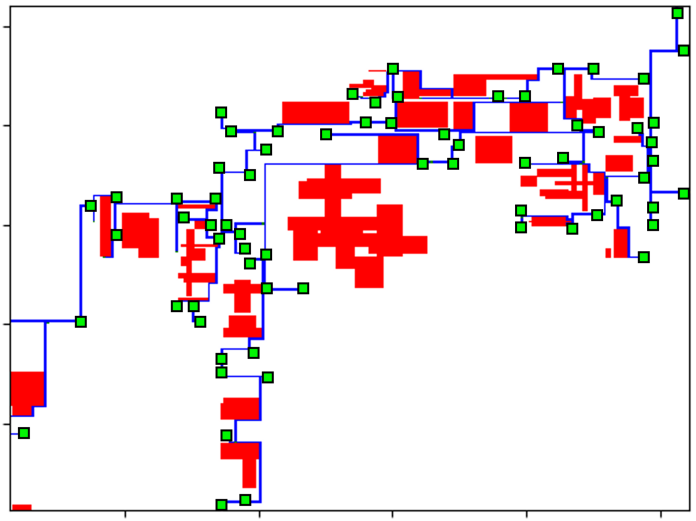

(c)

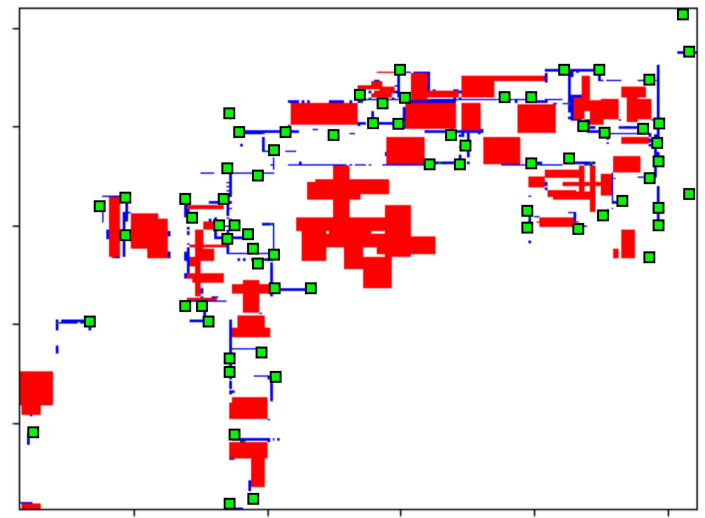

(b)

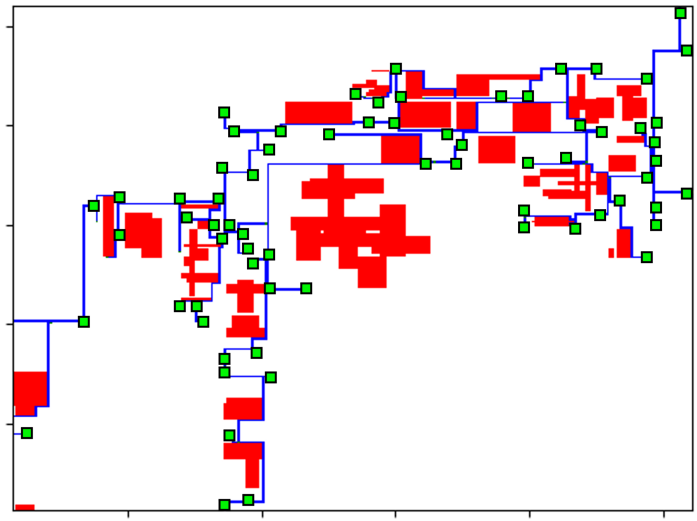

(d)

Fig. 11: ML outputs on different stages of model training. Terminals (enlarged for visibility), obstacles, and routed paths are shown in, respectively, green, red, and blue shades. Models at early training stages ((a) and (b)) produces invalid noisy outputs. Alternatively, a converged model produces a valid output with the same training set (c), similar to the optimally routed path (d).

tal algorithm (FLUTE) which lies at the core of other stateof-the-art pathfinders and is re-executed for a fare, hardwarespecific comparison. Alternatively, FOARS cannot be conveniently re-evaluated. Thus, performance metric from the original paper [4] are considered for FOARS comparison with the cGAN pathfinder. Note, however, that the frequency of the CPUs used for pathfinding evaluation in the original and this paper is comparable.

With the modern hardware accelerators, such as GPU, TPU, or NPU, batching individual ML inference requests can significantly impact ML runtime and throughput performance [25]. While the optimal batching parameters vary for different models, systems, and environments, the throughput-to-latency ratio can usually be efficiently controlled by batching within hardware constraints (e.g., batch data should fit into hardware accelerator memory). In practical applications such as IC design, millions of pathfinding tasks are solved during each pathfinding iteration. Thus, pathfinding throughput (as determined by the number of solved pathfinding tasks per unit of time) is a critical metric and should be considered along with the traditional pathfinding runtime metric. To maximize throughput performance, a batch size of 16 samples is preferred, yielding a $5 \times$ higher throughput as compared with non-batched single pathfinding inference. To account for both the parallel hardware accelerator ML processing and sequential CPU postprocessing with the proposed approach, pathfinding throughput is determined as

$$
T P_{c G A N}=\frac{\text { BatchSize }}{R T_{M L}+\text { BatchSize } \times R T_{\text {Postprocess }}},
$$

where $R T_{M L}$ is the runtime to route BatchSize paths with ML cGAN model, and $R T_{\text {Postprocess }}$ is the postprocessing runtime per a single path. Alternatively, the throughput of the existing CPU based sequential approaches is determined as one over a single pathfinding runtime.

Note, that the proposed formulation of pathfinding as image translation enables parallelization of pathfinding with nonbranching computations, propagating the input through the directed acyclic graph of cGAN generator submodel layers. Thus, the runtime of the cGAN pathfinder is not a function of the number and configuration of terminals and obstacles. Intuitively, ML processing runtime is constant and defined by the ML model, underlying framework implementation, and hardware. Based on the experimental results, the ML processing runtime of the trained ML model executed on NVIDIA GTX1080 GPU is $\approx 0.4$ seconds. Note that the postprocessing runtime varies between 0.1 and 0.2 seconds for all the tested data and is a function of the ML model prediction quality. Thus, the postprocessing runtime can be reduced with 
additional training. Alternatively, the runtime with traditional approaches increases quadratically [4], [24] with the increasing number of obstacles and terminals.

The constant runtime of the ML model is experimentally verified on a synthetic test set of $601,024 \times 1,024$ unseen pathfinding tasks generated based on the proposed methodology (see Section III-A). The length of the synthetically generated paths is considered as the reference length in these experiments. The number of terminals among the test set paths ranges between 10 and 1,063. The total area occupied by obstacles ranges between $6.7 \%$ and $35.8 \%$. All the 60 test samples are routed with the cGAN pathfinder. The wirelength with cGAN is similar to the reference length and the runtime for all the paths varies between $0.4+0.1=0.5$ and $0.4+0.2=0.6$ seconds.

The cGAN pathfinder is further evaluated with standard RT benchmarks. The experimental results are listed in Table I. As expected, the speedup with cGAN pathfinder over sequential state-of-the-art continuously increases with the increasing number of terminals and obstacles. Similarly, the throughput gain with cGAN also increases in more complex pathfinding problems. The projection of these trends is shown in Figure 12 based on extrapolated results from Table I and [4]. The data is extrapolated as follows: (i) The reported worst-case complexity of traditional pathfinding algorithms is $O\left(n^{2}\right)$, where $n$ is the number of terminals or obstacle corners of the input. (ii) The throughput of these algorithms is approximated as a reciprocal function of runtime. The cGAN pathfinder outperforms the FOARS algorithm in terms of runtime and throughput (for $\approx n>10^{3}-10^{4}-$ a realistic number of terminals and obstacles in modern and future pathfinding tasks). The cGAN pathfinder outperforms the MLOARSMT algorithm by over an order of magnitude even in small pathfinding systems. As compared with the proposed method, the traditional pathfinders are less practical in tasks with high number of terminals and obstacles.

TABLE I: Pathfinding performance comparison between the proposed cGAN pathfinder (with postprocessing) and traditional pathfinders (ML-OARSMT [24] and FOARS [4]).

\begin{tabular}{|l|c|c|c|c|c|}
\hline Benchmark & $R T 01$ & $R T 02^{\prime}$ & $R T 03$ & $R T 04$ & $R T 05^{\prime}$ \\
\hline \hline Terminals & 10 & 50 & 100 & 100 & 200 \\
Obstacles & 500 & 500 & 500 & 1,000 & 2,000 \\
\hline \hline \multicolumn{5}{|l|}{ Wirelength (tiles) } \\
\hline$[24]$ & 2,267 & 5,871 & 8,363 & 10,306 & 5,468 \\
{$[4]$} & 2,119 & $\mathrm{n} / \mathrm{a}$ & 8,282 & 10,330 & $\mathrm{n} / \mathrm{a}$ \\
cGAN & 2,272 & 5,873 & 8,412 & 10,287 & 5,476 \\
\hline \hline \multicolumn{5}{|l|}{ Runtime (seconds) } \\
\hline$[24]$ & 4.3 & 5.4 & 7.0 & 40.0 & 174.5 \\
{$[4]$} & $<0.01$ & 0.02 & 0.03 & 0.06 & 0.15 \\
cGAN & 0.5 & 0.5 & 0.5 & 0.6 & 0.5 \\
\hline \hline Throughput (Pathfinding tasks per seconds) \\
\hline$[24]$ & 0.23 & 0.19 & 0.14 & 0.025 & 0.0057 \\
{$[4]$} & $>100$ & 50.0 & 33.3 & 16.6 & 6.7 \\
cGAN & 5.5 & 5.5 & 5.5 & 3.6 & 5.5 \\
\hline
\end{tabular}

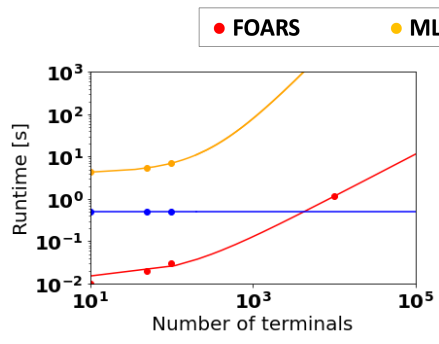

(a)

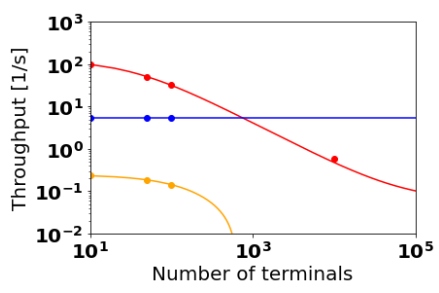

(c)

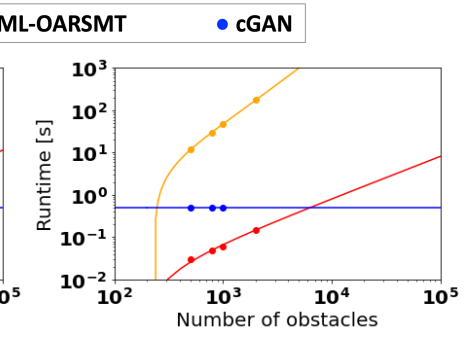

(b)

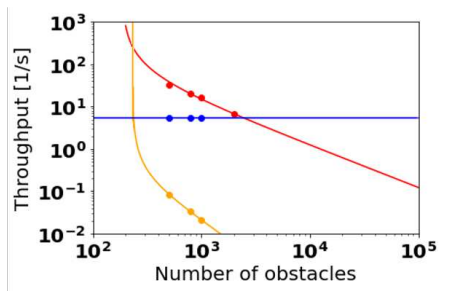

(d)
Fig. 12: Projection of multiterminal pathfinding performance with traditional state-of-the-art and proposed cGAN ML algorithms. Point markers represent existing data points. Curves represent the best fit functions: polynomial for runtime (to capture the quadratic scaling [4], [24]) and hyperbolic for throughput to capture the one over runtime behavior). Runtime as a function of terminal and obstacle count is shown in, respectively, (a) and (b). Throughput as a function of terminals and obstacles count is shown in, respectively, (c) and (d).

\section{CONClusion}

This work shows that a multiterminal obstacle avoiding pathfinding can be efficiently solved with a generative cGAN model executed on effective hardware accelerators, such as GPU, TPU, or NPU hardware. Based on the experimental results, the proposed cGAN model correctly determines paths in unseen benchmarks, yielding a state-of-the-art like path length and in those larger systems over an order of magnitude speedup and throughput gain. The proposed approach exploits the grid-like structure, that is most common in routing and navigation systems, to map the input pathfinding tasks and output paths to two-dimensional bitmaps and reduce the multiterminal obstacle-avoiding pathfinding to an image-toimage mapping. The proposed framework is enhanced with field-aware information and methodology for designing robust routed training dataset. Executing the pathfinding on parallel hardware accelerators allows to simultaneously and efficiently process high number of pathfinding tasks without additional overheads. The proposed cGAN pathfinding architecture and the methodology for designing synthetically-obtained training samples enables a fundamentally novel approach for obstacleavoiding multiterminal pathfinding in modern computing systems. This approach is expected to overcome some of the existing CPU bottlenecks by utilizing GPU or other parallel processing hardware. In particular, cGAN pathfinder is effective in industrial IC physical design tasks such as global routing and placement, as well as in autonomous vehicle navigation and planning. 


\section{REFERENCES}

[1] Jin Hu, Jarrod A Roy, and Igor L Markov, "Completing high-quality global routes," Proceedings of the 19th international symposium on Physical design. ACM, pp. 35-41, 2010.

[2] Huang-Yu Chen, Chin-Hsiung Hsu, and Yao-Wen Chang, "Highperformance global routing with fast overflow reduction," 2009 Asia and South Pacific Design Automation Conference. IEEE, pp. 582-587, 2009.

[3] Chris Chu, "FLUTE: fast lookup table based wirelength estimation technique," Proceedings of the 2004 IEEE/ACM International conference on Computer-aided design. IEEE Computer Society, pp. 696-701, 2004.

[4] Gaurav Ajwani, Chris Chu, and Wai-Kei Mak, "FOARS: FLUTE Based Obstacle-Avoiding Rectilinear Steiner Tree Construction," IEEE Transactions on Computer-Aided Design of Integrated Circuits and Systems, Vol. 30, No. 2, pp. 194-204, 2011.

[5] Zhonghua Zhou, Ziran Zhu, Jianli Chen, Yuzhe Ma, Bei Yu, Tsung-Yi Ho, Guy Lemieux, and Andre Ivanov, "Congestion-aware Global Routing using Deep Convolutional Generative Adversarial Networks," 2019 ACM/IEEE 1st Workshop on Machine Learning for CAD (MLCAD). IEEE, pp. 1-6, 2019.

[6] Zhiyao Xie, Yu-Hung Huang, Guan-Qi Fang, Haoxing Ren, Shao-Yun Fang, Yiran Chen, and Jiang $\mathrm{Hu}$, "RouteNet: Routability prediction for mixed-size designs using convolutional neural network," 2018 IEEE/ACM International Conference on Computer-Aided Design (IC$C A D)$. IEEE, pp. 1-8, 2018.

[7] Wei-Ting J Chan, Pei-Hsin Ho, Andrew B Kahng, and Prashant Saxena, "Routability optimization for industrial designs at sub- $14 \mathrm{~nm}$ process nodes using machine learning," Proceedings of the 2017 ACM on International Symposium on Physical Design, pp. 15-21, 2017.

[8] Wei-Ting J Chan, Yang Du, Andrew B Kahng, Siddhartha Nath, and Kambiz Samadi, "BEOL stack-aware routability prediction from placement using data mining techniques," 2016 IEEE 34th International Conference on Computer Design (ICCD). IEEE, pp. 41-48, 2016.

[9] Siting Liu, Qi Sun, Peiyu Liao, Yibo Lin, and Bei Yu, "Global placement with deep learning-enabled explicit routability optimization," IEEE/ACM Proceedings Design, Automation and Test in Eurpoe (DATE), 2021.

[10] Chak-Wa Pui, Gengjie Chen, Yuzhe Ma, Evangeline FY Young, and Bei Yu, "Clock-aware ultrascale fpga placement with machine learning routability prediction," 2017 IEEE/ACM International Conference on Computer-Aided Design (ICCAD). IEEE, pp. 929-936, 2017.

[11] Aysa Fakheri Tabrizi, Logan Rakai, Nima Karimpour Darav, Ismail Bustany, Laleh Behjat, Shuchang Xu, and Andrew Kennings, "A machine learning framework to identify detailed routing short violations from a placed netlist," 2018 55th ACM/ESDA/IEEE Design Automation Conference (DAC). IEEE, pp. 1-6, 2018.

[12] Cunxi Yu and Zhiru Zhang, "Painting on placement: Forecasting routing congestion using conditional generative adversarial nets," Proceedings of the 56th Annual Design Automation Conference 2019, pp. 1-6, 2019.

[13] Ilgweon Kang, Dongwon Park, Changho Han, and Chung-Kuan Cheng, "Fast and precise routability analysis with conditional design rules," Proceedings of the 20th System Level Interconnect Prediction Workshop, pp. 1-8, 2018

[14] Dani Maarouf, Abeer Alhyari, Ziad Abuowaimer, Timothy Martin, Andrew Gunter, Gary Grewal, Shawki Areibi, and Anthony Vannelli, "Machine-learning based congestion estimation for modern FPGAs," 2018 28th International Conference on Field Programmable Logic and Applications (FPL). IEEE, pp. 427-4277, 2018.

[15] Dmitry Utyamishev and Inna Partin-Vaisband, "Late Breaking Results: A Neural Network that Routes ICs," 2020 57th ACM/IEEE Design Automation Conference (DAC). IEEE, pp. 1-2, 2020.

[16] Keren Zhu, Mingjie Liu, Yibo Lin, Biying Xu, Shaolan Li, Xiyuan Tang, Nan Sun, and David Z Pan, "Geniusroute: A new analog routing paradigm using generative neural network guidance," 2019 IEEE/ACM International Conference on Computer-Aided Design (ICCAD). IEEE, pp. 1-8, 2019.

[17] Haiguang Liao, Wentai Zhang, Xuliang Dong, Barnabas Poczos, Kenji Shimada, and Levent Burak Kara, "A deep reinforcement learning approach for global routing," Journal of Mechanical Design, Vol. 142, No. 6, 2020.

[18] Sambhav R Jain and Kye Okabe, "Training a fully convolutional neural network to route integrated Circuits," arXiv preprint arXiv:1706.08948, 2017.

[19] Azalia Mirhoseini, Anna Goldie, Mustafa Yazgan, Joe Wenjie Jiang, Ebrahim Songhori, Shen Wang, Young-Joon Lee, Eric Johnson, Omkar Pathak, Azade Nazi, Jiwoo Pak, Andy Tong, Kavya Srinivasa, William Hang, Emre Tuncer, Quoc V. Le, James Laudon, Richard Ho, Roger
Carpenter, and Dean Jeff, "A graph placement methodology for fast chip design," Nature, Vol. 27, pp. 207-212, 2021.

[20] Adam Lerer, Ledell Wu, Jiajun Shen, Timothee Lacroix, Luca Wehrstedt, Abhijit Bose, and Alex Peysakhovich, "Pytorch-biggraph: A large-scale graph embedding system," arXiv preprint arXiv:1903.12287, 2019.

[21] Aditya Deshpande, Jiajun Lu, Mao-Chuang Yeh, Min Jin Chong, and David Forsyth, "Learning diverse image colorization," Proceedings of the IEEE Conference on Computer Vision and Pattern Recognition, pp. 6837-6845, 2017.

[22] Ruxin Wang and Dacheng Tao, "Non-local auto-encoder with collaborative stabilization for image restoration," IEEE Transactions on Image Processing, Vol. 25, No. 5, pp. 2117-2129, 2016

[23] Olaf Ronneberger, Philipp Fischer, and Thomas Brox, "U-net: Convolutional networks for biomedical image segmentation," International Conference on Medical image computing and computer-assisted intervention. Springer, pp. 234-241, 2015.

[24] Chung-Wei Lin, Shih-Lun Huang, Kai-Chi Hsu, Meng-Xiang Lee, and Yao-Wen Chang, "Multilayer obstacle-avoiding rectilinear Steiner tree construction based on spanning graphs," IEEE Transactions on Computer-Aided Design of Integrated Circuits and Systems, Vol. 27, No. 11, pp. 2007-2016, 2008.

[25] Christopher Olston, Noah Fiedel, Kiril Gorovoy, Jeremiah Harmsen, Li Lao, Fangwei Li, Vinu Rajashekhar, Sukriti Ramesh, and Jordan Soyke, "Tensorflow-serving: Flexible, high-performance ml serving," arXiv preprint arXiv:1712.06139, 2017. 